\title{
Roles of the Primate Motor Thalamus in the Generation of Antisaccades
}

\author{
Jun Kunimatsu ${ }^{1}$ and Masaki Tanaka ${ }^{1,2}$ \\ ${ }^{1}$ Department of Physiology, Hokkaido University School of Medicine, Sapporo 060-8638, Japan, and 2Precursory Research for Embryonic Science and \\ Technology, Japan Science and Technology Agency, Tokyo 102-0075, Japan
}

In response to changes in our environment, we select from possible actions depending on the given situation. The underlying neural mechanisms for this flexible behavioral control have been examined using the antisaccade paradigm. In this task, subjects suppress saccades to the sudden appearance of visual stimuli (prosaccade) and make a saccade in the opposite direction. Because recent imaging studies showed enhanced activity in the thalamus and basal ganglia during antisaccades, we hypothesized that the corticobasal ganglia loop may be involved. To test this, we recorded from neurons in the paralaminar part of the ventroanterior (VA), ventrolateral (VL) and mediodorsal (MD) nuclei of the thalamus when 3 monkeys performed pro/antisaccade tasks. For many VL and some VA neurons, the firing rate was greater during anti- than prosaccades. In contrast, neurons in the MD thalamus showed much variety of responses. For the population as a whole, neuronal activity in the VA/VL thalamus was strongly enhanced during antisaccades compared with prosaccades, while activity in the MD nucleus was not. Inactivation of the VA/VL thalamus resulted in an increase in the number of error trials in the antisaccade tasks, indicating that signals in the motor thalamus play roles in the generation of antisaccades. Enhancement of firing modulation during antisaccades found in the thalamus and those reported previously in the supplementary eye field and the basal ganglia suggest a strong functional linkage between these structures. The neuronal processes through the thalamocortical pathways might be essential for the volitional control of saccades.

\section{Introduction}

The ability to alter the stimulus-response association depending on a given situation is essential for the volitional control of movements. To investigate the underlying neural mechanisms, many previous studies used the antisaccade paradigm (Hallett, 1978). In this task, subjects are instructed to suppress eye movements toward a peripheral visual stimulus (prosaccade) and to generate a saccade in the opposite direction (antisaccade). The previous studies showed that the subjects with a variety of neurological or psychiatric disorders, including attention deficit hyperactivity disorder, schizophrenia, Parkinson's disease and Huntington's disease, had difficulties in the generation of antisaccades, suggesting that the corticosubcortical networks may be involved (for review, see Munoz and Everling, 2004).

Neuronal activity during antisaccades has been examined in many cortical and subcortical areas in monkeys. Many neurons in the supplementary eye field (SEF, Schlag-Rey et al., 1997) and the basal ganglia [caudate nucleus, Ford and Everling, 2009; Watanabe and Munoz, 2009; substantia nigra pars re-

Received Jan. 25, 2010; revised Feb. 17, 2010; accepted Feb. 25, 2010.

This work was supported by grants from the Japan Science and Technology Agency and the Ministry of Education, Culture, Sports, Science and Technology of Japan. We thank A. Yoshida, J. Tsunada, A. Matsushima and other members of our laboratory for their valuable comments and encouragement; T. Shiraishi and S. Hirano for their assistance in animal care, surgery, and histological work; and M. Suzuki for her administrative help. One monkey was provided by the Primate Research Institute of Kyoto University.

Correspondence should be addressed to Dr. Masaki Tanaka, Department of Physiology, Hokkaido University School of Medicine, North 15, West 7, Sapporo 060-8638, Japan. E-mail: masaki@med.hokudai.ac.jp.

DOI:10.1523/JNEUROSCI.0406-10.2010

Copyright $\odot 2010$ the authors $\quad 0270-6474 / 10 / 305108-10 \$ 15.00 / 0$ ticulata (SNr), Gore et al., 2005; globus pallidus (GP), Yoshida and Tanaka, 2009] showed greater firing modulation during antisaccades compared with prosaccades, whereas those in the frontal eye field (FEF, Everling and Munoz, 2000), the lateral intraparietal area (LIP, Gottlieb and Goldberg, 1999; Zhang and Barash, 2000) and the superior colliculus (SC, Everling et al., 1999) showed less activity for antisaccades than prosaccades. Thus, the task preference was different between many neurons in the basal ganglia and the SC, although the direct $\mathrm{SNr}-\mathrm{SC}$ projection has proven to be important for the generation of purposeful saccades (for review, Hikosaka et al., 2000). These results suggest that some of the signals in the basal ganglia might also regulate eye movements through the thalamocortical pathways.

Anatomically, the basal ganglia and the cerebellum send signals to the oculomotor areas in the frontal cortices via the multiple nuclei in the thalamus (Alexander et al., 1986; Lynch et al., 1994; Middleton and Strick, 2000). For example, neurons in the paralaminar part of the ventrolateral (VL) and ventroanterior (VA) nuclei mediate signals from the GP and $\mathrm{SNr}$ to the SEF, and those in the adjacent mediodorsal (MD) nucleus receive signals from the SC and $\mathrm{SNr}$ and send outputs to the SEF and FEF (for review, see Lynch and Tian, 2006). Consistent with the thalamic involvement in saccade generation, many neurons in the thalamus carry saccade-related signals (VA/VL, Schlag-Rey and Schlag, 1984; Wyder et al., 2003; MD, Tanibuchi and Goldman-Rakic, 2003), and recent studies showed that signals through the motor thalamus regulate the timing of self-initiated saccades (Tanaka, 2006, 2007). Furthermore, several imaging studies have revealed enhanced ac- 


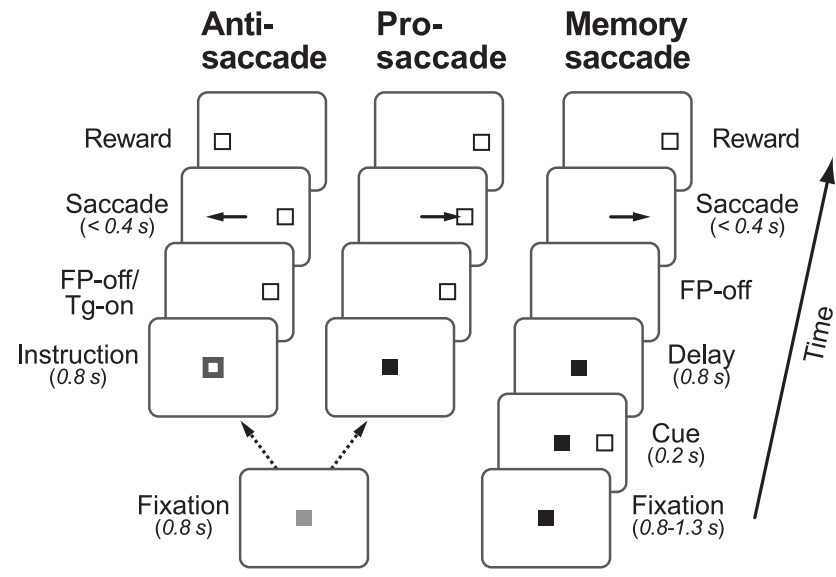

Figure 1. Sequence of events in the three saccade tasks. In both the antisaccade task and the prosaccade task, color of the fixation point was initially gray, and it became green (antisaccade task) or red (prosaccade task) for $800 \mathrm{~ms}$. A white target spot appeared $16^{\circ}$ eccentrically at the time of the fixation point offset. Monkeys were required to make a saccade toward (prosaccade) or away from (antisaccade) the target within $400 \mathrm{~ms}$. In the memory saccade task, color of the fixation point was red throughout the trial. A peripheral target flashed briefly $(200 \mathrm{~ms})$ during central fixation, and monkeys were required to maintain fixation and remember the location of the target. After a delay of $800 \mathrm{~ms}$, the fixation point disappeared and monkeys made a saccade to the location of the previously flashed target within $400 \mathrm{~ms}$. Different conditions were randomly interleaved within a block.

tivity during antisaccades in the thalamus (O'Driscoll et al., 1995; Matsuda et al., 2004).

Considering these observations, we hypothesized that the corticosubcortical network through the motor thalamus play roles in the control of antisaccades. To test this, we examined the activity of single thalamic neurons and the effects of local inactivation. We found that many neurons showed enhanced activity during antisaccades, and signals through the motor thalamus indeed play a causal role in the generation of antisaccades.

\section{Materials and Methods}

Three Japanese monkeys (Macaca fuscata, D, male $14 \mathrm{~kg}$; E and N, females $6 \mathrm{~kg}$ ) were used. All animal protocols were approved in advance by the Hokkaido University Animal Care and Use Committee, and were in accordance with the Guide for the Care and Use of Laboratory Animals (National Research Council, 1996). The experimental procedures were identical to those described previously (Tanaka, 2005; Yoshida and Tanaka, 2009).

Animal preparation. Initially, monkeys were habituated to sitting in a primate chair. The animals were implanted with a pair of head holders and an eye coil, using sterile procedures under general halothane anesthesia. Following the recovery from the surgery, monkeys were trained on the oculomotor tasks. During the training and the subsequent experimental sessions, the monkey's head was secured to the primate chair in a darkened booth, and horizontal and vertical eye position signals were recorded using the search coil technique (MEL-25; Enzanshi Kogyo). After training on eye movement tasks, a second surgery was undertaken to place a recording cylinder for vertical electrode penetration aimed at the thalamus. Daily recording sessions began after full recovery from the surgery. Water intake was controlled daily so that monkeys were motivated to perform the behavioral tasks.

Visual stimuli and behavioral tasks. Experiments were controlled by a Windows-based real-time data acquisition system (TEMPO; Reflective Computing) running on Pentium PCs. Visual stimuli were presented on a 24 inch cathode-ray tube monitor (refresh rate: $60 \mathrm{~Hz}$ ) that was located $38 \mathrm{~cm}$ away from the eyes, and subtended to $64 \times 44^{\circ}$ of the visual angle. Three saccade paradigms were used; antisaccade, prosaccade and memory saccade tasks (Fig. 1). Each trial began with the appearance of a fixation point $\left(0.5^{\circ}\right.$ square spot $)$ at the center of the screen. In both the prosaccade task and the antisaccade task, the fixation point was initially gray for $800 \mathrm{~ms}$, and monkeys were required to maintain eye position within $2^{\circ}$ of the fixation point. Then, the color of the fixation point was changed either to red or green for $800 \mathrm{~ms}$ to instruct monkeys of the trial type (Instruction period). When the color of the fixation point was red, monkeys were required to make a saccade to the target that appeared at the end of the instruction period (prosaccade). When the fixation point became green, monkeys had to make a saccade with the same amplitude as the target eccentricity but in the opposite direction (antisaccade). In both tasks, the fixation point was extinguished at the end of the instruction period, and the target was presented $16^{\circ}$ eccentrically in one of 8 predetermined directions ( $45^{\circ}$ apart starting with the right direction). In the antisaccade task, the target was relocated to the opposite side and at the same eccentricity after 350-400 ms of its appearance. Monkeys were required to move their eyes to the 'window' that surrounded the target location 350-400 ms after the fixation point offset. The size of the window was $6^{\circ}$ for the antisaccade task and $3^{\circ}$ for the prosaccade task. In the memory saccade task, the red fixation point appeared on the screen. After a random $800-1300 \mathrm{~ms}$ fixation interval, a white target was presented for $200 \mathrm{~ms}$ ("cue"). Monkeys were required to remember its location and to maintain fixation for an additional $800 \mathrm{~ms}$. Once the fixation point disappeared, monkeys had to make a saccade to the location of the previously presented target within $400 \mathrm{~ms}$. The target reappeared $400 \mathrm{~ms}$ following the fixation point offset, and monkeys were required to maintain fixation ( $4^{\circ}$ window) for an additional $800 \mathrm{~ms}$. The insertion of the second fixation interval was useful to dissociate the times of saccades and rewards. Correct performance was reinforced with a drop of liquid reward at the end of each trial. Each trial was presented in a pseudorandom order within a block that usually consisted of 6 different trials (3 different tasks in opposite directions).

Single neuron recording and local inactivation. To record from single neurons, a tungsten electrode (FHC Inc.) was lowered into the thalamus though the 23-gauge guide tube using a micromanipulator (MO-97S; Narishige). The location of each penetration was adjusted using the $x-y$ stage or the grid system (Crist Instruments) attached to the cylinder. The guide tube was advanced $13-15 \mathrm{~mm}$ from the surface of the intact dura. For most experiments, the dorsal surface of the thalamus could be identified rather easily because of the existence of the callosal fibers and the lateral ventricle. Signals obtained from the electrodes were amplified (Model 1800; A-M Systems), filtered (Model 3625; NF Co.), and monitored online using oscilloscopes and an audio device. We isolated the waveforms of a single neuron using a real time spike sorter with templatematching algorithms (MSD; Alpha Omega Engineering). The occurrence of action potentials was time stamped and saved in files with the data of eye movements and visual stimuli during experiments.

We searched for the task-related neurons when monkeys performed a block of 10 randomized conditions consisting of the memory saccade trials in 8 different directions and horizontal antisaccade trials (all presented $16^{\circ}$ eccentrically). Once a single thalamic neuron was isolated, we determined the optimal eye movement direction during experiments by analyzing the data for the memory saccade trials. We then configured the block of saccade trials to include tasks only along the preferred axis for the neuron under study. For approximately one-third of saccade-related neurons that did not show any clear directional preference, neuronal activity was examined during horizontal eye movements.

After the recording experiments were terminated, the effects of local inactivation were examined in three hemispheres of two monkeys ( $D$ and N). A 28-gauge injection needle (Crist Instruments) connected to a $10 \mu \mathrm{l}$ Hamilton microsyringe was inserted through the guide tube, and a small amount of $\mathrm{GABA}_{\mathrm{A}}$ agonist (muscimol hydrobromide; $5.0 \mu \mathrm{g} / \mu \mathrm{l}$ dissolved in saline, volume $1.0 \mu \mathrm{l}$ ) was pressure-injected around the recording sites at a rate of $\sim 0.2 \mu \mathrm{l} / 30 \mathrm{~s}$. The monkey performed a block of 6 randomized conditions consisting of the pro/anti and memory saccade trials in both horizontal directions. The effect of inactivation was assessed by comparing eye movements before and 10-75 min after injection. We also performed the control experiments by injecting saline $(1.0 \mu \mathrm{l})$ into the effective sites in separate sessions.

Histological procedures. Recording sites were reconstructed from the histological sections of two animals (monkeys D and E). At the end of the 
experiments, several electrolytic lesions were made at or near the sites where the task-related neurons were recorded. Lesions were made by passing a direct current $(10-20 \mu \mathrm{A})$ through the recording electrodes for $30-40 \mathrm{~s}$. The animals were then deeply anesthetized with sodium pentobarbital ( $>50 \mathrm{mg} / \mathrm{kg}$, i.p.) and perfused with $0.1 \mathrm{~m}$ phosphate buffer followed by $3.5 \%$ formalin. The brain was cut into coronal sections at $50 \mu \mathrm{m}$ thicknesses using a freezing microtome, and each section was stained using the Nissl method (cresyl violet). The location of each task-related neuron was reconstructed according to the depth and the coordinates of electrode penetrations and the relative locations of the marking lesions. The third animal (monkey $\mathrm{N}$ ) is still in use for another project, and the locations of electrode penetrations for this animal are shown in supplemental Figure $3 A$ (available at www. jneurosci.org as supplemental material). For this animal, neurons located $\geq 4 \mathrm{~mm}$ lateral to the midline were regarded as VA/VL neurons, while those located within $3.5 \mathrm{~mm}$ from the midline were regarded as MD neurons. The properties of neuronal firing for each group in this animal were similar to those in the other two animals (supplemental Figs. 3B, 4, available at www.jneurosci.org as supplemental material).

Data acquisition and analyses. The data of spike timing and eye movements were digitized and sampled at $1 \mathrm{kHz}$, and were stored in files during the experiments. Further off-line analyses were performed using Matlab (MathWorks). In some antisaccade trials, monkeys initially generated a saccade toward the target, and redirected their eyes to the location opposite to the target within $400 \mathrm{~ms}$ ("turn-around saccade"). Since we detected error trials by analyzing eye position before and $400 \mathrm{~ms}$ (or 350 $\mathrm{ms}$ in some cases) after the fixation point offset during experiments, monkeys were rewarded in such trials. We detected the turn-around saccades off-line and considered them as errors. While these trials were excluded from the analyses of neuronal activity described below, they were included when we counted the number of error trials before and after thalamic inactivation (see Figs. 6, 7).

Neuronal activity was measured during the following three time periods: 1) the $400 \mathrm{~ms}$ interval immediately before the cue or the instruction (baseline period), 2) the $200 \mathrm{~ms}$ period preceding the fixation point offset (delay or instruction period), and 3) the $120 \mathrm{~ms}$ interval starting from $100 \mathrm{~ms}$ before saccades in the optimal direction that showed the largest activity during memory saccades (saccade period). In this study, we considered neurons that exhibited a significant firing modulation during the 3 task intervals (one-way ANOVA, $p<0.05$ ). We defined as 'saccaderelated' when the neuron showed differential activity between the baseline period and the saccade period according to a post hoc multiple comparison in any task ( $p<0.05$, Scheffé). Similarly, we considered as 'instruction-related' for the neuron showing a differential activity between the baseline period and the instruction period.

To assess the effects of thalamic inactivation, we computed the rate of error trials before and after inactivation for each condition. During experiments, monkeys sometimes aborted trials by looking away from the fixation point before the target appearance. The trials with early fixation break were repeated later in the block to obtain a balanced number of trials for each condition. Because we were interested in the roles of the thalamus in the generation of goal-directed behavior, the error rates were computed only for trials in which the monkey maintained fixation until the fixation point disappeared. For the memory saccade task, the proportion of error trials with an early saccade toward the cue was computed separately to quantify the effects of thalamic inactivation.

\section{Results}

\section{Basic properties of neuronal activity in the thalamus}

We searched for neurons that discharged before saccades in the paralaminar part of the VL, VA, and MD nuclei of the thalamus in 4 hemispheres of 3 monkeys. Among 210 task-related neurons, 136 were recorded from VA/VL nuclei ( 89 from monkey D, 21 from monkey E and 26 from monkey N) and 74 were recorded from MD nucleus (50 from monkey D and 24 from monkey N). Data obtained from the VA/VL nuclei were examined separately from those obtained from the MD nucleus. Since we were sometimes unable to reliably determine the lateral border of the intralaminar nuclei on the histological sections, a few neurons likely to be located within the intralaminar nuclei were regarded as VA/VL neurons (Fig. 2). In addition, because we were unable to obtain histological sections from the third animal (monkey N, supplemental Fig. 3A, available at www.jneurosci.org as supplemental material) and because it was sometimes difficult to determine the border between the VA nucleus and the area X of the VL thalamus on each histological section, we regarded neurons located laterally as VA/VL, or motor thalamic, neurons. Based on 

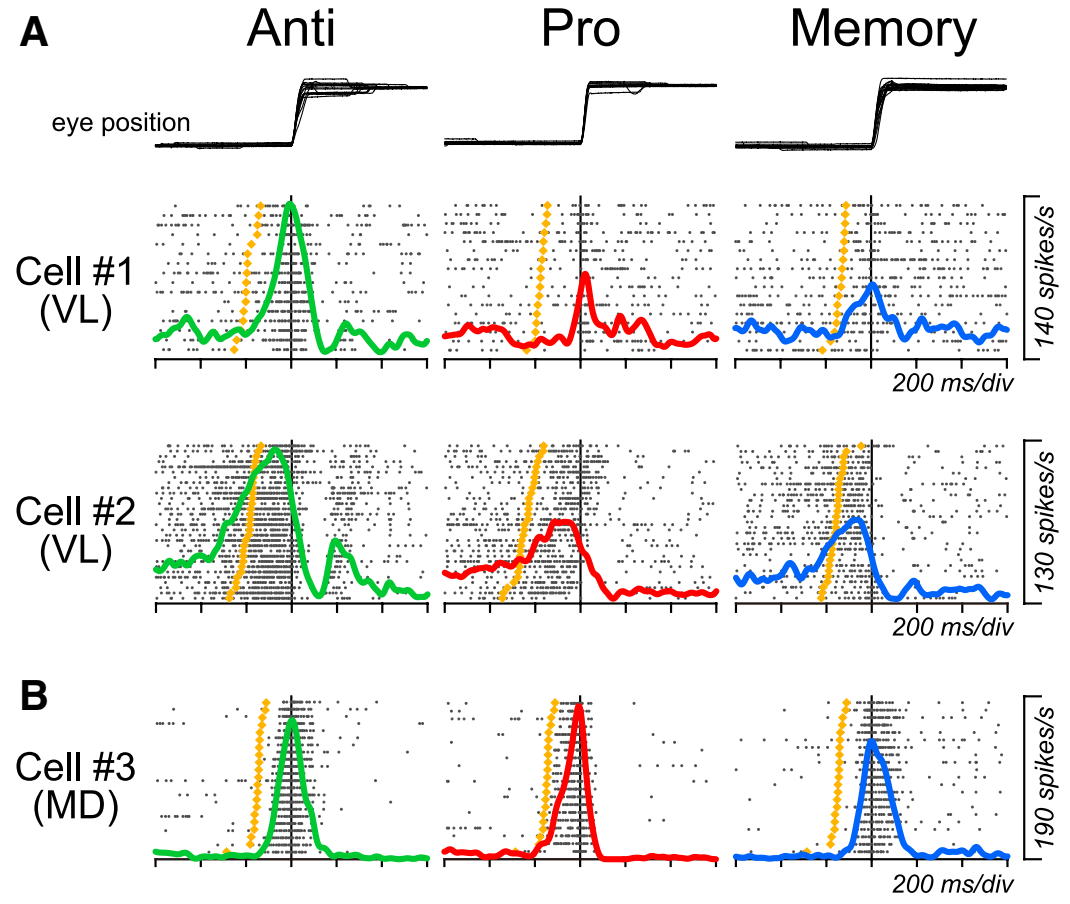

Figure 3. Comparison of neuronal activity between three saccade paradigms. $\boldsymbol{A}$, Two examples of VL neurons. $\boldsymbol{B}, \mathrm{An}$ MD neuron. In all panels, data are aligned on saccade initiation (vertical line). Trials are sorted by saccade latency, and the rasters and the corresponding spike density are shown for saccades in the preferred direction only. The yellow dot on each raster line indicates the time of the fixation point offset.

histological examination in two monkeys, only 5 neurons were located definitely within the VA nucleus (Fig. 2). The numbers of neurons in different nuclei and hemispheres are summarized in supplemental Table 1, available at www.jneurosci.org as supplemental material.

To characterize the difference in neuronal activity between VA/VL and MD thalamus, we first examined the directional preference of each neuron by having monkeys perform memory saccades in 8 different directions. The directional tuning of the saccade-related activity was assessed by fitting a Gaussian curve (least squares, $r^{2}>0.7$ ) to the firing rates for trials in different directions (supplemental Fig. 1, available at www.jneurosci.org as supplemental material). Among 195 neurons formally tested, the preferred directions could be determined reliably for 138 neurons (VA/VL, $n=81 / 125,65 \%$; MD, $n=57 / 70,81 \%$ ). The proportion of directional neurons and the distribution of the preferred directions were similar to those reported previously (supplemental Fig. $2 \mathrm{~A}$, available at www.jneurosci.org as supplemental material) (VA/VL, Wyder et al., 2003; MD, Tanibuchi and Goldman-Rakic, 2003; Watanabe and Funahashi, 2004). The preferred directions of the VA/VL neurons were uniformly distributed, whereas those of the MD neurons showed a slight but statistically significant bias toward the contraversive saccades (Rayleigh test, $p<0.05$; supplemental Fig. $2 B$, available at www. jneurosci.org as supplemental material). These findings were also consistent with previous reports, suggesting that the sites of our recordings were comparable to those of the previous studies.

We next examined the neuronal modulation during the three saccade paradigms. A hundred sixty task-related neurons were recorded during anti/pro- and memory saccade tasks along the preferred axis for each neuron (Materials and Methods). Almost all neurons exhibited differential activity between the baseline period and the saccade period (Scheffé, $p<0.05$; VA/VL, $n=$ 95/99; $\mathrm{MD}, n=61 / 61$ ), and approximately half of them showed differential activity between the baseline period and the instruction period $(p<$ 0.05; VA/VL, $n=45 / 99 ; \mathrm{MD}, n=25 / 61)$. Supplemental Figure $2 C$ (available at www.jneurosci.org as supplemental material) compares the mean firing rates of the VA/VL neurons with those of the MD neurons during the three saccade tasks in the opposite directions. In the population, the baseline activity of the VA/VL neurons was greater than that of the MD neurons (unpaired $t$ test, $p<$ 0.01 ), while the size of the saccaderelated transient tended to be larger for the MD neurons than for the VA/VL neurons. These differential properties were also found when the data from the third animal (monkey N), from which we were unable to obtain histological sections, were plotted separately (supplemental Fig. 3B, available at www. jneurosci.org as supplemental material). During recordings, these different firing properties between the nuclei were useful to determine the locations of individual neurons, along with the stereotaxic coordinates of the electrode penetrations. In the following sections, we will describe the properties of the saccade-related activity first and then consider the instruction-period activity.

\section{Comparison of the saccade-related activities across the paradigms}

Many saccade-related neurons in the VA/VL, or motor, thalamus exhibited greater firing modulation during antisaccades than during the other two tasks. Figure 3 plots the data from three representative neurons for saccades in the preferred direction. The neuron in the top row showed a burst of activity just before contraversive saccades. The saccade-related neuron in the middle row also exhibited a gradual buildup of activity during the preparation for saccade. For both VL neurons in Figure 3A, the activity was much greater for antisaccades than the other two tasks. In contrast, an example neuron in Figure $3 B$ was recorded from the MD thalamus and showed a similar amount of activity across the paradigms.

To quantify the saccade-related activities, the firing rates of individual neurons were measured in a $120 \mathrm{~ms}$ window starting from $100 \mathrm{~ms}$ before saccades in the preferred direction. Figure $4 \mathrm{~A}$ compares the firing rates during antisaccades with those during prosaccades for individual neurons in the motor thalamus. A one-way ANOVA with a post hoc multiple comparisons revealed that the firing rates of $37 \%$ (35/95) neurons were statistically different between the tasks (Scheffé, $p<0.05$, filled circles in Fig. $4 A$ ). For the population of VA/VL neurons, the activity during antisaccades was statistically different from that during prosaccades (paired $t$ test, $p<0.01$; prosaccade: $33.5 \pm 22.4$ spikes/s, antisaccade: $41.5 \pm 26.2$ spikes/s). The time courses of the population activity for the 95 saccade-related neurons in the VA/VL thalamus (Fig. $4 \mathrm{~B}$ ) again confirmed that the activity during antisaccades was greater than that during prosaccades. As for the 61 saccade-related neurons in the MD, 33 (54\%) showed activity that was different between the tasks (Scheffé, $p<0.05$, filled circles in Fig. 4C). Twenty neurons (33\%) exhibited sig- 
nificantly greater activity during antisaccades than prosaccades, whereas 13 neurons $(21 \%)$ exhibited less activity during antisaccades than prosaccades. For the population of MD neurons, the firing rate during antisaccades was not statistically different from that during prosaccades (paired $t$ test, $p>0.05$; prosaccade: $41.8 \pm 29.5 \mathrm{spikes} / \mathrm{s}$, antisaccade: $47.2 \pm 28.3$ spikes/s). The time courses of the population activity in Figure $4 D$ also display a similar amount of activity for the tasks. Thus, neurons in the motor thalamus showed enhanced activity during antisaccades, whereas those in the MD nucleus displayed a variety of responses during saccades in different tasks. This tendency was also found when the data from the third monkey was separately analyzed (supplemental Fig. 4, available at www. jneurosci.org as supplemental material). To summarize the population activity during anti/prosaccades in opposite directions, supplemental Figure $2 C$ (available at www. jneurosci.org as supplemental material) plots the mean firing rates for all taskrelated neurons. Regardless of the type of saccades, the activity during saccades in the preferred direction (defined by the activity during memory saccades) was consistently greater than that for saccades in the opposite direction (unpaired $t$ test, $p<0.01$ ), indicating that no reversal of the preferred direction occurred during antisaccades.

\section{Preparatory activity during the}

\section{instruction period}

For each trial, monkeys were required to alter eye movement responses to the peripheral target depending on the color of the fixation point. We next examined whether the thalamic neurons exhibited firing modulation during saccade preparation depending on the paradigms. For this purpose, we selected neurons that showed differential activity between the baseline period and the end of the instruction period that was $200 \mathrm{~ms}$ before the fixation point offset (Scheffé, $p<0.05$, Materials and Methods). Among 160 task-related neurons, 70 exhibited the preparatory activity (VA/VL, $n=45 / 99,47 \%$; MD, $n=25 / 61,41 \%$ ) in either (VA/VL, $n=4$; MD, none) or both saccade paradigms (VA/VL, $n=41 ; \mathrm{MD}, n=25$ ).

Figure $5 \mathrm{~A}$ shows a representative VL neuron with the instruction-related activity. Because the saccade target appeared at the time of the fixation point offset, no directional information was available during the instruction period. Therefore, the data for trials in opposite directions were combined to examine neuronal activity during the instruction period. The neuron in Figure $5 \mathrm{~A}$ showed a greater instruction-period activity for antisaccades than prosaccades. To quantify the modulation of the instruction-period activity for individual neurons, we measured the firing rates during the $200 \mathrm{~ms}$ immediately before the fixation point offset. Among 45 VA/VL neurons, 19 (42\%) displayed the instruction-period activity that was statistically differ- ent between the paradigms (unpaired $t$ test, $p<0.05$; filled circles in Fig. $5 B$ ). For the population of neurons, the instruction-period activity during the antisaccade task was significantly different from that in the prosaccade task (paired $t$ test, $p<0.01$; prosaccade: $22.3 \pm 16.9$ spikes/s, antisaccade: $27.4 \pm 21.1$ spikes/s). The time courses of the population activity plotted in Figure $5 \mathrm{C}$ show that the task-dependent firing modulation emerged $\sim 400 \mathrm{~ms}$ following the instruction and was evident at the end of the $800 \mathrm{~ms}$ instruction interval. For the MD neurons with the instruction-period activity, $48 \%(n=12 / 25)$ showed differential activity between the tasks (Fig. $5 D$, filled circles). For the population of the MD neurons, however, the firing rate during the preparation for antisaccades was not statistically different from that during the preparation for prosaccades (paired $t$ test, $p>0.1$; prosaccade: $17.9 \pm 13.3$ spikes/s, antisaccade: $20.2 \pm 12.5$ spikes/s), whereas the time courses of the population activity plotted in Figure $5 E$ exhibit a slight difference between the tasks.

To exclude the possibility that the differential activity during saccade preparation was related to the color selectivity of thalamic neurons, we examined the activity of $7 \mathrm{VL}$ neurons during a block of the fixation trials. In this task, the color of the fixation point changed as in the antisaccade task (that is, from gray to green), but the saccade target never appeared and the fixation point remained. Although the instruction-period activity in the 
A

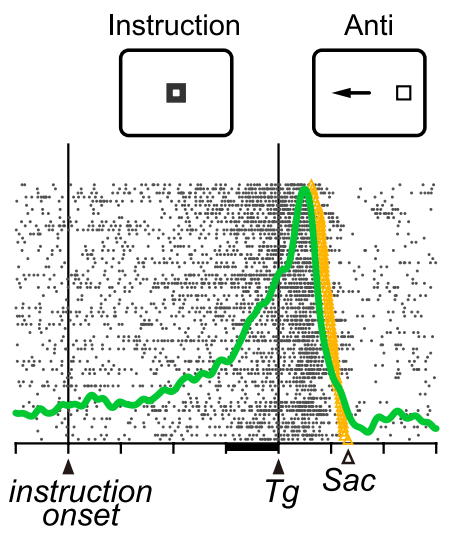

B

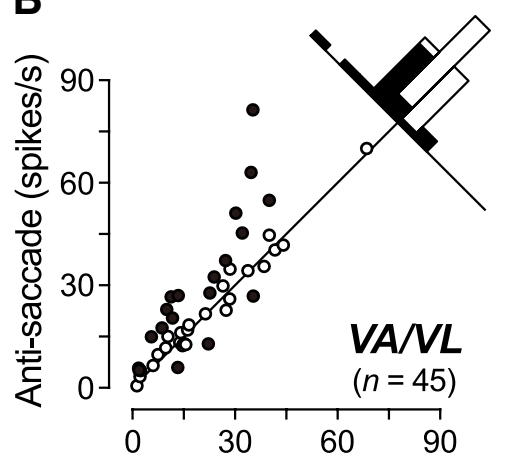

Pro-saccade (spikes/s)

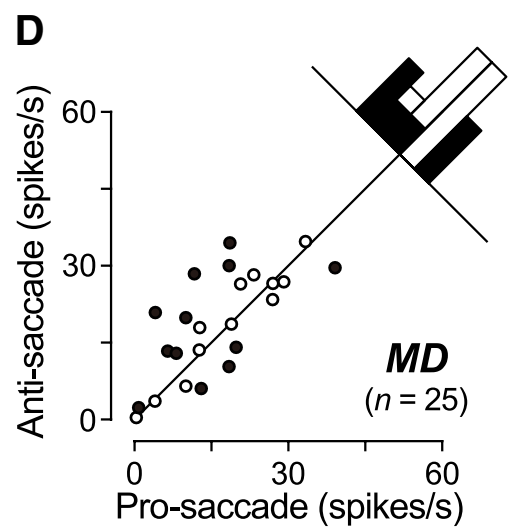

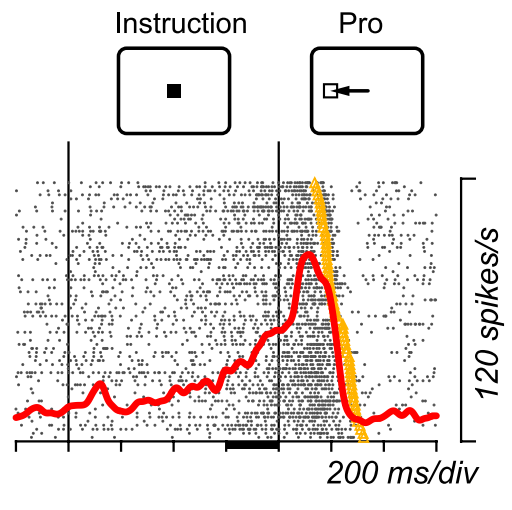

C

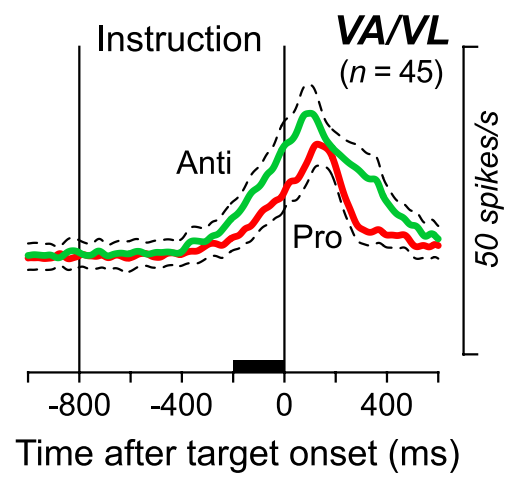

E

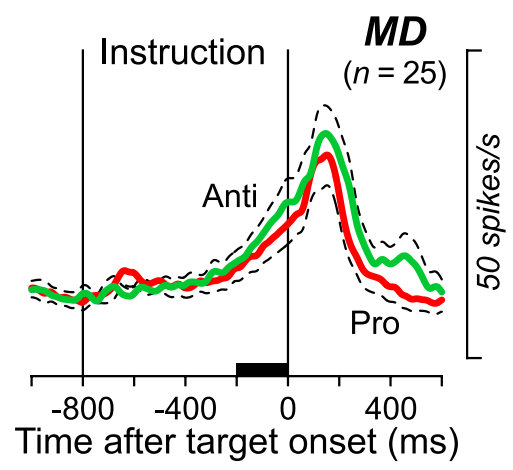

Figure 5. Task-dependent firing modulation during the instruction period. $\boldsymbol{A}$, An example VL neuron showing differential activity during the instruction period. Color of the fixation point was different between the two saccade tasks during the period delimited by two vertical lines (Instruction period). The target appeared at the time of the fixation point offset (second vertical line). To examine the activity during the instruction period, data for saccades in opposite directions were combined. The yellow dot on each raster line indicates the time of saccade. The colored traces indicate spike densities computed from the associated rasters. $\boldsymbol{B}, \boldsymbol{D}$, The instruction period activities are compared between the antisaccade trials and the prosaccade trials for individual neurons recorded from the VA/VL $(\boldsymbol{B})$ and the MD $(\boldsymbol{D})$ nuclei. The firing rate was measured for a $200 \mathrm{~ms}$ interval immediately before the target onset (black bars in $\boldsymbol{A}, \boldsymbol{C}$, and $\boldsymbol{E}$ ). Filled symbols represent the data showing a significant difference between the tasks (two-tailed $t$ test, $p<0.05$ ). One outlier point has been omitted from each of the scatter plots in $\boldsymbol{B}([81,97])$ and $\boldsymbol{C}([60,56])$ but is included in the histogram. $\boldsymbol{C}, \boldsymbol{E}$, Time courses of the population activity for neurons in the VA/VL $(\boldsymbol{C})$ and the MD $(\boldsymbol{E})$ nuclei. The traces are the means of spike densities computed for individual neurons. Dashed traces indicate plus or minus SEM.

antisaccade trials was statistically different from the baseline (paired $t$ test, $p<0.01$, difference in activity, $27.2 \pm 15.0$ spikes $/ \mathrm{s}$, $n=7$ ), the firing modulation greatly reduced during the block of the fixation trials $(5.3 \pm 4.1$ spikes/s), suggesting that neuronal activity during the instruction period was context-dependent, likely related to the preparation for antisaccades.

\section{Effects of local inactivation}

To explore the causal roles of the signals in the thalamus in the generation of antisaccades, the recording sites were reversibly inactivated with a small amount of muscimol ( $1 \mu \mathrm{l}$ in volume, concentration $5 \mu \mathrm{g} /$ $\mu \mathrm{l})$ in 3 hemispheres in two monkeys (D and $\mathrm{N})$. Sites of inactivation were selected from those containing the task-related neurons found in the separate experiments. Postmortem histological examinations in monkey $\mathrm{D}$ revealed that the sites of inactivation were in the VA $(n=1), \mathrm{VL}$ $(n=5)$ and $\mathrm{MD}(n=5)$ nuclei (Fig. 2; supplemental Table 2, available at www. jneurosci.org as supplemental material). For monkey N, we attempted to inactivate 3 sites in the VA/VL nuclei and 2 sites in the MD nucleus (supplemental Fig. 3A, available at www.jneurosci.org as supplemental material). Figure 6 plots the data obtained from one experiment (site \#3). Even before inactivation, the monkey made erroneous prosaccades in 5\% of antisaccades trials (upper row, red traces). During inactivation in the right motor thalamus, the error rate increased significantly in the leftward, contraversive antisaccade trials $\left(55 \%, \chi^{2}\right.$ test, $\left.p<0.01\right)$. In many of the error trials, the monkey redirected his eyes away from the target even before the target was relocated $400 \mathrm{~ms}$ after the fixation point offset in the antisaccade task (Materials and Methods). On the other hand, the error rate remained unchanged both in the prosaccade trials and the memory saccade trials, whereas the monkey sometimes made an early reflexive saccade toward the cue in the memory saccade task during inactivation. Such trials were not counted in computing the rate of the error trials here but will be considered later (Materials and Methods, see the next but one paragraph and supplemental Table 2, available at www. jneurosci.org as supplemental material).

Figure $7 A$ summarizes the changes in error rate following muscimol $(n=16)$ or saline $(n=4)$ injection. For 9 inactivation experiments performed in the motor thalamus, the error rate increased dramatically in the antisaccade trials (Fig. 7A, data connected with red lines), whereas the performance of prosaccades remained unchanged. In 5 of these 9 experiments, the antisaccade deficits were found only contralaterally, while the inactivation effects were found bilaterally in the remaining experiments. Injections of saline into the effective sites (asterisks in Fig. 2 and supplemental Fig. $3 A$, available at www.jneurosci.org as supplemental material) in the motor thalamus did not alter the error rates in either task (Fig. 7A, black dashed lines). For inactivation of the MD thalamus, the error rate in all saccade paradigms re- 
mained unchanged in 4 of 7 experiments, while the error rate of contraversive antisaccades increased modestly in 3 experiments (Fig. 7A, blue triangles). For only one $\mathrm{MD}$ site $(\# 12)$, we also found a slight but statistically significant $(p<0.01)$ change in error rate in the contraversive prosaccade task in which the monkey made erroneous antisaccades. Thus, inactivation of the VA/VL thalamus consistently altered the antisaccade performance, and the effects were found bilaterally in approximately half of the experiments. In contrast, inactivation of the MD nucleus failed to alter antisaccade performance in most experiments. Although the existence of early corrective saccades in the erroneous antisaccade trials (Fig. 6) suggested that inactivation of the motor thalamus likely facilitated automatic reflexive saccades, the reaction time of erroneous prosaccades was comparable to that of correct prosaccades in most experiments (supplemental Fig. 5, available at www.jneurosci.org as supplemental material).

The modest inactivation effect found in some MD experiments might be attributed to the spread of muscimol into the motor thalamus. Figure $7 B$ plots the time courses of the changes in error rate in the contraversive antisaccade trials for all 9 inactivation experiments performed in the motor thalamus (red lines) and for the 3 effective experiments in the MD nucleus (blue lines). The error rates were computed for every $900 \mathrm{~s}$ intervals centered at the data points (separated by $450 \mathrm{~s}$ ), and the black circles plot the means $( \pm \mathrm{SD})$ of the data from the VA/VL experiments. The error rate increased gradually $20-30 \mathrm{~min}$ after injection, indicating that the muscimol effects were due to GABAergic inhibition, but not to the immediate volume effects on nearby structures. Interestingly, the inactivation effects in the MD experiments emerged later than those in most of the VA/VL experiments, suggesting that the inactivation effects in the MD experiments might be due to the spread of muscimol into the motor thalamus.

Since we were interested in the thalamic roles in the generation of saccades, the trials with an early fixation break before the fixation point offset were not considered to compute the error rates described above. During experiments, such trials were aborted and repeated later within the block of trials to obtain a balanced number of trials across the paradigms (Materials and Methods). Because the target appeared at the time of the fixation point offset in both the pro/ antisaccade tasks, the early saccades in the pro/antisaccade tasks did not provide any information about flexible control of goaldirected behavior. In contrast, the occurrence of early saccades test, $\left.{ }^{* *} p<0.01\right)$.

C
Anti Pro Memory ipsi target contra target
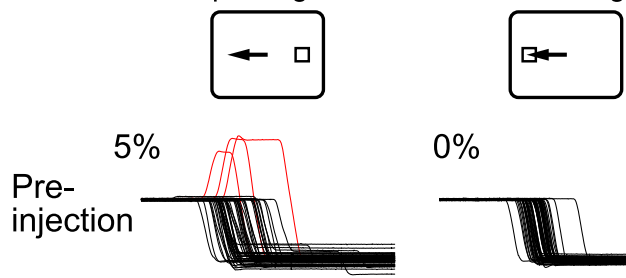

$0 \%$

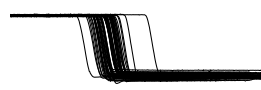

$0 \%$ contra target

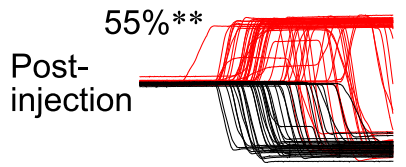

$0 \%$

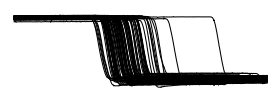

$0 \%$
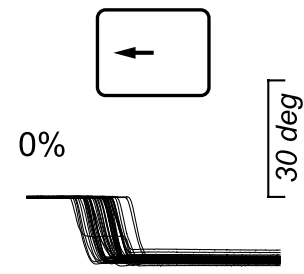

Figure 6. Effects of local inactivation on saccades. Traces indicate horizontal eye position in the antisaccade (left), prosaccade (middle), and memory saccade (right) paradigms before and after muscimol injection (1 $\mu$ l, right VL, site\#3). Data are aligned with the offset of the fixation point. Red traces indicate error trials. The number on each panel denotes the proportion of error trials. $\left(\chi^{2}\right.$
A

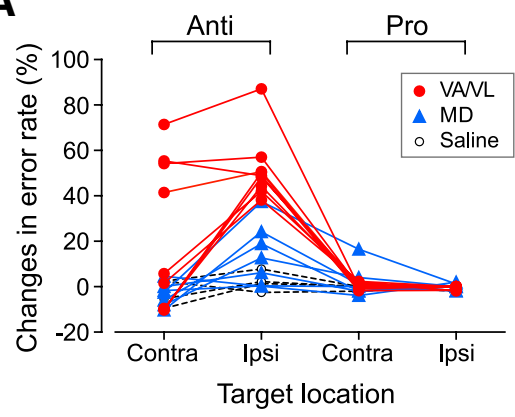

B

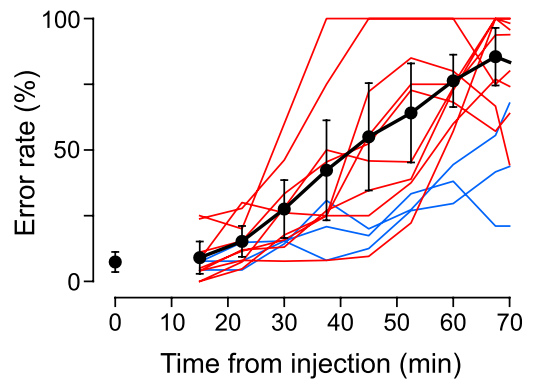

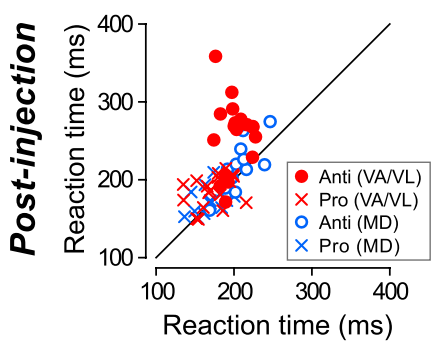
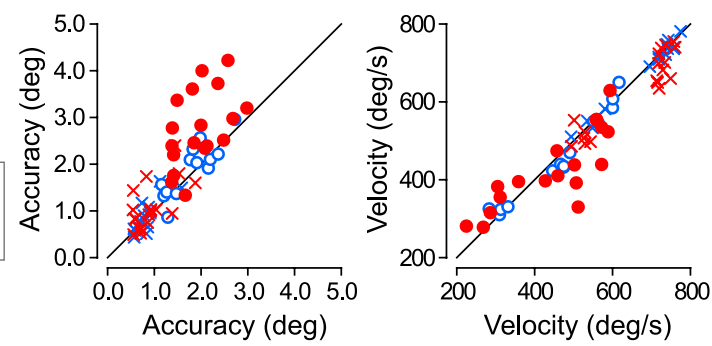

Pre-injection

Figure 7. Quantification of the inactivation effects. $A$, Changes in error rate for all injection experiments. Data of muscimol experiments in the VA/VL and MD nuclei are plotted by red and blue symbols, respectively. Data for saline injections in the motor thalamus is connected by black dashed lines. $\boldsymbol{B}$, Time courses of the error rates after muscimol injections. The error rates of contraversive antisaccades were computed during every 900 s intervals (separated by $450 \mathrm{~s}$ ) following muscimol injection for all 9 VA/VL experiments (red lines) and 3 MD experiments (blue lines). Black dots indicate the means of the VA/VL data. Error bar indicates the $95 \%$ confidence interval. The error rate during the final $900 \mathrm{~s}$ in the preinjection block is plotted at time 0 . C, Comparison of the saccade parameters before and during inactivation. On each panel, the circles indicate the data for correct antisaccades in both directions. The $X$ s indicate the data for prosaccades. Different colors denote different nuclei in the thalamus. toward the cue before the fixation point offset in the memory saccade task may reflect the impairment of behavioral suppression. Supplemental Table 2 summarizes the rate of error trials with early reflexive saccades during the inactivation experiments. On average, the reflexive saccades toward the cue in the memory saccade task were observed in $2 \pm 2 \%$ of trials before inactivation 
(ranged from 0 to $7 \%, n=32$ cases), while they became $9 \pm 12 \%$ (ranged from 0 to $44 \%, n=18$ cases) and $1 \pm 2 \%$ (ranged from 0 to $7 \%, n=14$ cases) following inactivation of the VA/VL and MD nuclei, respectively. For individual experiments, the proportion of reflexive saccades toward the cue increased significantly ( $\chi^{2}$ test, $p<0.01$ ) in 3 of $9 \mathrm{VA} / \mathrm{VL}$ experiments (all bilateral sites) and none for the MD experiments. Except for one of the four VA/VL experiments, early reflexive saccades were also observed for trials in both directions, suggesting that some signals in the motor thalamus might affect cortical processing bilaterally. For the 3 effective VA/VL sites, the latency of early saccades toward the cue during inactivation averaged $216 \pm 13 \mathrm{~ms}(\mathrm{SD}$, ranged from 200 to $231 \mathrm{~ms}, n=5$ cases). The increased number of memory saccade trials with an early fixation break implies that one of the roles of the motor thalamus in antisaccades might be to suppress unwanted prosaccades.

A previous study in our laboratory showed that inactivation in the external segment of the GP (GPe) modestly increased the error rate of antisaccades, but the saccade parameters in successful antisaccade trials during inactivation were generally normal (Yoshida and Tanaka, 2009). Conversely, we found in this study that inactivation of the motor thalamus not only increased the error rate, but also affected the parameters of correct antisaccades. Figure $7 C$ compares the saccadic reaction time, accuracy and peak velocity of correct anti/prosaccades in both directions before and after muscimol injection into the thalamus. Inactivation of the motor thalamus prolonged the latency (paired $t$ test, $p<0.01)$ and worsened the accuracy $(p<0.01)$ of antisaccades (red circles), while neither the latency nor the accuracy of prosaccades was altered $(p>0.05)$. A reduction of peak velocity was found in prosaccades only $(p<0.01)$, whereas one monkey showed a significant reduction of antisaccade velocity during $\mathrm{VA} / \mathrm{VL}$ inactivation (monkey $\mathrm{D}, p<0.05$ ). Inactivation of the MD nucleus did not alter any saccade parameter during both pro/antisaccade tasks ( $p>0.05$, blue symbols). Thus, the signals in the motor thalamus play a prominent role in the generation of antisaccades, whereas the roles of the MD nucleus in this context are uncertain.

\section{Discussion}

This study showed for the first time that the motor thalamus is involved in the generation of antisaccades. Neurons in the paralaminar part of the VA/VL thalamus exhibited a significant firing modulation during both the preparation and execution of antisaccades. We also found that the occurrence of erroneous saccades in antisaccade trials dramatically increased following inactivation of the motor thalamus. In contrast, inactivation of the MD thalamus did not show any consistent effect. The motor thalamus may play roles in antisaccades by relaying signals from the basal ganglia to the cortex and by regulating the local processing between the thalamus and the cortex.

\section{Enhancement of neuronal firing during antisaccades}

The previous functional imaging studies have revealed the enhanced activity in many cortical and subcortical areas during antisaccades compared with prosaccades (for humans, Matsuda et al., 2004; Tu et al., 2006; for monkeys, Ford et al., 2009). However, direct analyses of individual neuronal firing in monkeys have shown that the magnitude of firing modulation in these structures was not necessarily enhanced during antisaccades, possibly because the blood-oxygen-level-dependent signal reflects both the synaptic potentials (Logothetis et al., 2001; Viswanathan and Freeman, 2007) and the non-neuronal vascular
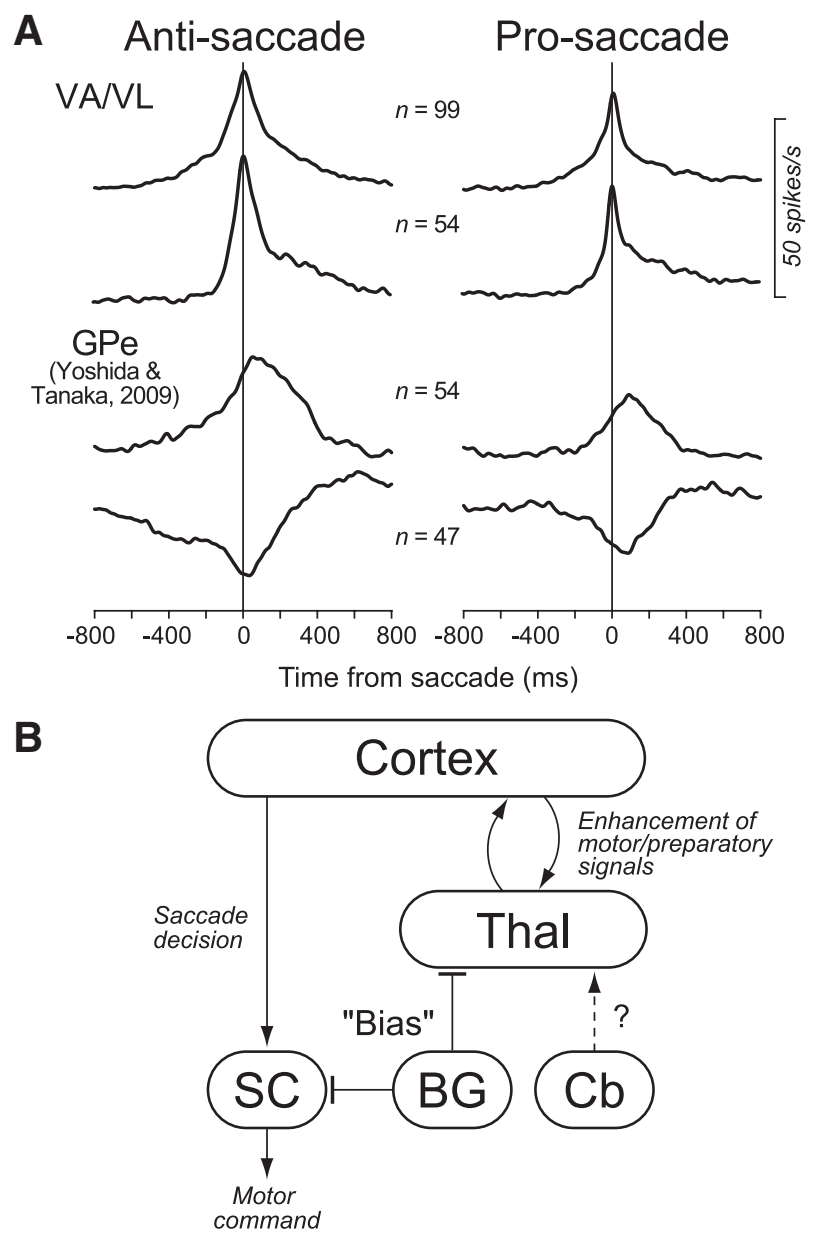

Figure 8. A, Comparison of the population activity for neurons in the motor thalamus and the GPe. The data for anti/prosaccades are shown separately in different columns. On each column, the top trace shows the population activity computed for all task-related neurons recorded from the motor thalamus. The second trace was computed only for the neurons in the motor thalamus that lacked a significant firing modulation during the instruction period. The bottom two traces plot the data of GPe neurons that exhibited either increased (third trace) or decreased (fourth trace) firing rate during saccades obtained in the previous study (Yoshida and Tanaka, 2009). B, A possible neural mechanism of the basal ganglia-thalamocortical pathways in the generation of antisaccades. In this diagram, the motor drive and preparatory signals for antisaccades are processed within the mutual thalamocortical projections, while the basal ganglia regulate the gain of this intrinsic pathway. The arrows and bars indicate excitatory and inhibitory connections, respectively. BG, Basal ganglia; Cb, cerebellum; Thal, thalamus.

elements that can change in a few seconds (Sirotin and Das, 2009). Indeed, the task-related neurons in the lateral prefrontal cortex (PFC, Everling and DeSouza, 2005), FEF (Everling and Munoz, 2000), LIP (Gottlieb and Goldberg, 1999; Zhang and Barash, 2000) and the anterior cingulate cortex (ACC, Johnston et al., 2007) generally exhibit less or a similar amount of activity during antisaccades compared with prosaccades. Similarly, the magnitude of the presaccadic burst of activity in the SC is also smaller during antisaccades than during prosaccades (Everling et al., 1999).

In contrast, we found in this study that neuronal activity in the motor thalamus was strongly enhanced during antisaccades. For the population of VA/VL neurons, the firing rate immediately before and during antisaccades was statistically greater than that during prosaccades. The previous studies reported that, like neurons in the motor thalamus, many neurons in the SEF (SchlagRey et al., 1997; Amador et al., 2004), the basal ganglia (Ford and 
Everling, 2009; Watanabe and Munoz, 2009; Yoshida and Tanaka, 2009), as well as the SC projecting neurons in the lateral PFC (Johnston and Everling, 2006) exhibit greater firing modulation during antisaccades than prosaccades. The enhancement of the firing modulation in the motor thalamus, SEF, PFC, and the basal ganglia suggests a strong functional linkage between these regions in the control of antisaccades.

Roles of the motor thalamus in the generation of antisaccades Anatomically, the motor thalamus transmits signals from the basal ganglia and the cerebellum to many cortical areas including the SEF, FEF and PFC (Tian and Lynch, 1997; Middleton and Strick, 2000). Therefore, the enhanced signals during antisaccades may be processed serially through the corticobasal gangliathalamocortical loop, consisting of the SEF, the basal ganglia and the motor thalamus. Figure $8 \mathrm{~A}$ compares the time courses of the population activity for neurons in the motor thalamus (this study) and in the GPe (Yoshida and Tanaka, 2009) obtained from the same animals. Neurons in both structures showed a prelude activity during saccade preparation, and the firing modulation around the time of saccades was greater during antisaccades than prosaccades. However, the time course of the saccade-related transient was somewhat different between these structures. Neurons in the motor thalamus exhibited a brisk response, and the peak of the transient was close to the time of saccade initiation. This was clearly observed when the population activity was computed only for neurons that lacked the instruction period activity (55\%, Fig. $8 \mathrm{~A}$, second traces). On the other hand, the peak of the firing modulation for the GPe neurons lagged behind saccade initiation, and the activity lasted for $>400 \mathrm{~ms}$ (Fig. $8 \mathrm{~A}$, bottom two traces). The time course of Gpi (internal segment of the GP) neurons was similar to that of the GPe neurons (data not shown). Although the clipped activity observed in thalamic neurons could attribute to the local inhibitory feedback from the reticular nucleus, the difference in the properties of the saccade-related transient between the thalamus and the GPe suggests that the saccade-related signal in the thalamus may not be solely inherited from neuronal activity in the basal ganglia, or alternatively, that neurons in the other basal ganglia structures such as the $\mathrm{SNr}$ might exhibit more brisk response.

Because inactivation of the motor thalamus (this study) or the GPe (Yoshida and Tanaka, 2009) resulted in an increase of erroneous prosaccades in the antisaccade paradigms, one of the roles of these structures appears to be the suppression of unnecessary prosaccades. During inactivation of the motor thalamus, the impairments were sometimes found bilaterally (Fig. 7A). The nondirectional deficits might be attributed to the loss of the enhanced signals during the instruction period. Alternatively, the transient signals before saccades in the population of VA/VL neurons could also aid in the suppression of unwanted saccades in both directions, because one third of saccade-related neurons were nondirectional and the preferred directions of the remaining neurons distributed all directions (supplemental Fig. 2, available at www.jneurosci.org as supplemental material). In addition to the changes in the error rate, we also found the effects on saccade parameters. During inactivation of the motor thalamus, the reaction time of correct antisaccades was prolonged and the accuracy decreased in almost all experiments, whereas inactivation of the GPe did not show any consistent effect on saccade parameters in the previous study (Yoshida and Tanaka, 2009). These results suggest that the motor thalamus is located within the pathways that regulate the antisaccade motor commands, while the GPe may not.
To explain these observations, we propose the model outlined in Figure $8 B$. In this diagram, the transient signals during antisaccades in the motor thalamus come from the cortex, and the local thalamocortical loop enhances this signal. The basal ganglia regulate the gain of the thalamocortical processing by modulating the strength of their inhibitory outputs to the thalamus. The basic idea of this hypothesis is essentially the same as the well established view on the roles of the nigrocollicular pathways in saccade generation (Hikosaka and Wurtz, 1985; Hikosaka et al., 2000). In the original idea, the motor drive in the SC is thought to be provided directly from the cortex, and the inhibitory pathway through the SNr serves as a "permissive gating" to allow for collicular neurons to gain access to the cortical signals. Similarly, the basal ganglia might regulate the intrinsic gain control mechanisms between the thalamus and the cortex, rather than relaying the transient or motor drive signals, for the generation of voluntary eye movements (cf. Nambu, 2008). For example, the excitatory signals in the GPe shown in Figure $8 \mathrm{~A}$ might facilitate the thalamocortical processing by inhibiting neurons in the GPi or $\mathrm{SNr}$, while the inhibitory signals in the GPe could suppress another specific thalamocortical processing through the crossed or uncrossed projections to the thalamus. The mutual connections between the motor thalamus and the frontal cortex are known to exist (McFarland and Haber, 2002), and the importance of the thalamocortical reciprocal pathways for the cortical sensory processing is well documented (Guillery and Sherman, 2002). However, we realize that additional experiments are needed to verify this hypothesis. In particular, we are aware that our recording sites in the rostral part of the paralaminar VL thalamus receive inputs from both the basal ganglia and the cerebellum, but the projection from the latter is dominant (DeVito and Anderson, 1982; Anderson and Turner, 1991; Middleton and Strick, 2000). To our knowledge, however, there is no previous study suggesting the roles of the cerebello-thalamocortical pathways in antisaccades, except for a few imaging studies showing enhanced activity in the cerebellum (Luna et al., 2001; Tu et al., 2006).

\section{Roles of the signals in the mediodorsal thalamus}

Neurons in the MD thalamus exhibited a strong burst of activity before saccades, and for the population as a whole, the magnitude of activity was not statistically different between pro and antisaccades. Anatomical studies indicate that neurons in the MD nucleus relay signals from the SC and SNr to many cortical areas including the FEF, SEF and PFC (Tian and Lynch, 1997; Middleton and Strick, 2000). We found a variety of neurons in the MD thalamus with respect to the preference for anti or prosaccades (Fig. 4C). Neurons showing a strong preference for prosaccades might transmit signals from the SC to the cortex, while those showing an enhanced activity during antisaccades might receive inputs from the cortex and/or from the SNr. Inactivation of these neurons failed to alter antisaccade performance in 4 of 7 experiments, and the inactivation effects found in the remaining experiments were weak and started late (Fig. $7 A, B$ ). These results suggest that the signals in the MD thalamus are unlikely to participate in the generation of antisaccades. The lack of a contribution of the MD thalamus to the initiation of voluntary eye movements is consistent with the previous studies (Tanaka, 2006). The signals in the MD might be involved in the internal monitoring of saccades (Sommer and Wurtz, 2002), rather than their generation. 


\section{References}

Alexander GE, DeLong MR, Strick PL (1986) Parallel organization of functionally segregated circuits linking basal ganglia and cortex. Annu Rev Neurosci 9:357-381.

Amador N, Schlag-Rey M, Schlag J (2004) Primate antisaccade. II. Supplementary eye field neuronal activity predicts correct performance. J Neurophysiol 91:1672-1689.

Anderson ME, Turner RS (1991) Activity of neurons in cerebellar-receiving and pallidal-receiving areas of the thalamus of the behaving monkey. J Neurophysiol 66:879-893.

DeVito JL, Anderson ME (1982) An antoradiographic study of efferent connections of the globus pallidus in Macaca mulatta. Exp Brain Res 46:107-117.

Everling S, DeSouza JF (2005) Rule-dependent activity for prosaccades and antisaccades in the primate prefrontal cortex. J Cogn Neurosci 17:14831496.

Everling S, Munoz DP (2000) Neuronal correlates for preparatory set associated with pro-saccades and anti-saccades in the primate frontal eye field. J Neurosci 20:387-400.

Everling S, Dorris MC, Klein RM, Munoz DP (1999) Role of primate superior colliculus in preparation and execution of anti-saccades and prosaccades. J Neurosci 19:2740-2754.

Ford KA, Everling S (2009) Neural activity in primate caudate nucleus associated with pro- and antisaccades. J Neurophysiol 102:2334-2341.

Ford KA, Gati JS, Menon RS, Everling S (2009) BOLD fMRI activation for anti-saccades in nonhuman primates. Neuroimage 45:470-476.

Gore JL, Marino RA, Munoz DP (2005) Neural correlates associated with pro- and anti-saccades in primate SNr. Soc Neurosci Abstr 35:167.6.

Gottlieb J, Goldberg ME (1999) Activity of neurons in the lateral intraparietal area of the monkey during an antisaccade task. Nat Neurosci 2:906-912.

Guillery RW, Sherman SM (2002) Thalamic relay functions and their role in corticocortical communication: generalizations from the visual system. Neuron 33:163-175.

Hallett PE (1978) Primary and secondary saccades to goals defined by instructions. Vision Res 18:1279-1296.

Hikosaka O, Wurtz RH (1985) Modification of saccadic eye movements by GABA-related substances. II. Effects of muscimol in monkey substania nigra pars reticulata. J Neurophysiol 53:292-308.

Hikosaka O, Takikawa Y, Kawagoe R (2000) Role of the basal ganglia in the control of purposive saccadic eye movements. Physiol Rev 80:953-978.

Johnston K, Everling S (2006) Monkey dorsolateral prefrontal cortex sends task-selective signals directly to the superior colliculus. J Neurosci 26:12471-12478.

Johnston K, Levin HM, Koval MJ, Everling S (2007) Top-down controlsignal dynamics in anterior cingulate and prefrontal cortex neurons following task switching. Neuron 53:453-462.

Logothetis NK, Pauls J, Augath M, Trinath T, Oeltermann A (2001) Neurophysiological investigation of the basis of the fMRI signal. Nature 412:150-157.

Luna B, Thulborn KR, Munoz DP, Merriam EP, Garver KE, Minshew NJ, Keshavan MS, Genovese CR, Eddy WF, Sweeney JA (2001) Maturation of widely distributed brain function subserves cognitive development. Neuroimage 13:786-793.

Lynch JC, Tian JR (2006) Cortico-cortical networks and cortico-subcortical loops for the higher control of eye movements. Prog Brain Res 151: 461-501.

Lynch JC, Hoover JE, Strick PL (1994) Input to the primate frontal eye field from the substantia nigra, superior colliculus, and dentate nucleus demonstrated by transneuronal transport. Exp Brain Res 100:181-186.
Matsuda T, Matsuura M, Ohkubo T, Ohkubo H, Matsushima E, Inoue K, Taira M, Kojima T (2004) Functional MRI mapping of brain activation during visually guided saccades and antisaccades: cortical and subcortical networks. Psychiatry Res 131:147-155.

McFarland NR, Haber SN (2002) Thalamic relay nuclei of the basal ganglia form both reciprocal and nonreciprocal cortical connections, linking multiple frontal cortical areas. J Neurosci 22:8117-8132.

Middleton FA, Strick PL (2000) Basal ganglia and cerebellar loops: motor and cognitive circuits. Brain Res Brain Res Rev 31:236-250.

Munoz DP, Everling S (2004) Look away: the anti-saccade task and the voluntary control of eye movement. Nat Rev Neurosci 5:218-228.

Nambu A (2008) Seven problems on the basal ganglia. Curr Opin Neurobiol 18:1-10.

O’Driscoll GA, Alpert NM, Matthysse SW, Levy DL, Rauch SL, Holzman PS (1995) Functional neuroanatomy of antisaccade eye movements investigated with positron emission tomography. Proc Natl Acad Sci U S A 92:925-929.

Schlag-Rey M, Schlag J (1984) Visuomotor functions central thalamus in monkey. I. Unit activity related to spontaneous eye movements. J Neurophysiol 51:1149-1174.

Schlag-Rey M, Amador N, Sanchez H, Schlag J (1997) Antisaccade performance predicted by neuronal activity in the supplementary eye field. Nature 390:398-401.

Sirotin YB, Das A (2009) Anticipatory haemodynamic signals in sensory cortex not predicted by local neuronal activity. Nature 457:475-479.

Sommer MA, Wurtz RH (2002) A pathway in primate brain for internal monitoring of movements. Science 296:1480-1482.

Tanaka M (2005) Involvement of the central thalamus in the control of smooth pursuit eye movements. J Neurosci 25:5866-5876.

Tanaka M (2006) Inactivation of the central thalamus delays self-timed saccades. Nat Neurosci 9:20-22.

Tanaka M (2007) Cognitive signals in the primate motor thalamus predict saccade timing. J Neurosci 27:12109-12118.

Tanibuchi I, Goldman-Rakic PS (2003) Dissociation of spatial-, object-, and sound-coding neurons in the mediodorsal nucleus of the primate thalamus. J Neurophysiol 89:1067-1077.

Tian JR, Lynch JC (1997) Subcortical input to the smooth and saccadic eye movement subregions of the frontal eye field in Cebus monkey. J Neurosci 17:9233-9247.

Tu PC, Yang TH, Kuo WJ, Hsieh JC, Su TP (2006) Neural correlates of antisaccade deficits in schizophrenia, an fMRI study. J Psychiatr Res 40:606-612.

Viswanathan A, Freeman RD (2007) Neurometabolic coupling in cerebral cortex reflects synaptic more than spiking activity. Nat Neurosci 10: $1308-1312$.

Watanabe M, Munoz DP (2009) Neural correlates of conflict resolution between automatic and volitional actions by basal ganglia. Eur J Neurosci 30:2165-2176.

Watanabe Y, Funahashi S (2004) Neuronal activity throughout the primate mediodorsal nucleus of the thalamus during oculomotor delayed-responses. I. Cue-, delay-, and response-period activity. J Neurophysiol 92:1738-1755

Wyder MT, Massoglia DP, Stanford TR (2003) Quantitative assessment of the timing and tuning of visual-related, saccade-related, and delay period activity in primate central thalamus. J Neurophysiol 90:2029-2052.

Yoshida A, Tanaka M (2009) Enhanced modulation of neuronal activity during antisaccades in the primate globus pallidus. Cereb Cortex 19: 206-217.

Zhang M, Barash S (2000) Neuronal switching of sensorimotor transformations for antisaccades. Nature 408:971-975. 